PROFESSOR BRETT DELAHUNT (Orcid ID : 0000-0002-5398-0300)

MRS ALLISON STEIGLER (Orcid ID : 0000-0002-4775-1632)

PROFESSOR LARS EGEVAD (Orcid ID : 0000-0001-8531-222X)

Article type : Original Article

\title{
Perineural invasion by prostate adenocarcinoma in needle biopsies predicts bone metastasis: Ten year data from the TROG 03.04 RADAR Trial
}

B. Delahunt, ${ }^{1}$ J.D. Murray ${ }^{1}$, A. Steigler, ${ }^{2}$ C. Atkinson, ${ }^{3}$ D. Christie,${ }^{4}$ G. Duchesne,${ }^{5}$

L. Egevad, ${ }^{6}$ D. Joseph, ${ }^{7}$ J. Matthews,${ }^{8}$ C. Oldmeadow, ${ }^{9}$ H. Samaratunga, ${ }^{10}$ N.A. Spry, ${ }^{11}$ J.R

Srigley, ${ }^{12}$ H. Hondermarck, ${ }^{9,13}$ J.W. Denham ${ }^{2}$

${ }^{1}$ Department of Pathology and Molecular Medicine and Health Sciences, University of Otago, Wellington, New Zealand; ${ }^{2}$ School of Medicine and Public Health, University of Newcastle, Newcastle, New South Wales, Australia; ${ }^{3}$ St Georges Cancer Care Centre, Christchurch, New Zealand; ${ }^{4}$ Genesis Care, Tugan, Queensland, Australia; ${ }^{5}$ Peter MacCallum Cancer Centre and University of Melbourne, Victoria, Australia; ${ }^{6}$ Department of Oncology and Pathology, Karolinska Institute, Stockholm, Sweden; ${ }^{7}$ Sir Charles Gairdner Hospital, Perth, Western Australia, Australia; ${ }^{8}$ Auckland City Hospital, Auckland, New Zealand; ${ }^{9}$ Hunter Medical Research Institute, Newcastle, New South Wales, Australia; ${ }^{10}$ Aquesta Uropathology and University of Queensland, Brisbane, Queensland, Australia; ${ }^{11}$ GenesisCare, Perth, Western Australia; ${ }^{12}$ Department of Laboratory Medicine and Pathobiology and Molecular Medicine, University of Toronto, Toronto, ON, Canada, ${ }^{13}$ School of Biomedical Sciences and Pharmacy, University of Newcastle, New South Wales, Australia

This is the author manuscript accepted for publication and has undergone full peer review but has not been through the copyediting, typesetting, pagination and proofreading process, which may lead to differences between this version and the Version of Record. Please cite this article as doi: 10.1111/HIS.14107

This article is protected by copyright. All rights reserved 
Address for Correspondence: Professor Brett Delahunt, Department of Pathology and Molecular Medicine, University of Otago, P.O. Box 7343, Wellington South, New Zealand. E-mail: brett.delahunt@otago.ac.nz

Short Running Title: Perineural invasion predicts bone metastasis

Conflict of Interest Statement: The authors have no conflicts of interest to declare.

-

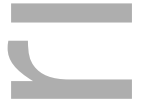

Trial number: The TROG 03.04 Trial is registered with the National Institutes of Health Clinical Trials Registry, number NCT00193856 and has approval from Hunter New England Human Research Ethics Committee (Trial ID. 03/06/11/3.02)

Word Count 2615 words

ABSTRACT

Aims: Perineural invasion (PNI) by prostatic adenocarcinoma is debated as a prognostic parameter. This study investigates the prognostic predictive value of PNI in a series of patients with locally advanced prostate cancer treated with radiotherapy and androgen deprivation using 10 year outcome data from the TROG 03.04 RADAR trial.

Methods: Diagnostic prostate biopsies from 976 patients were reviewed and the presence of PNI noted. Patients were followed for 10 years according to the trial protocol or until death. The primary endpoint for the study was time to bone metastasis. Secondary endpoints included time to soft tissue metastasis, transition to castration resistance, prostate cancerspecific mortality and all-cause mortality.

Results: PNI was detected in 449 cases (46\%), with 234 cases (24\%) having PNI in more than one core. The presence of PNI was significantly associated with higher ISUP grade, clinical T staging category, National Comprehensive Cancer Network risk group, and percent positive biopsy cores. The cumulative probability of bone metastases according to PNI status 
was significant over the 10 year follow-up interval of the study (log-rank test $p<0.0001)$. PNI was associated with all endpoints on univariable analysis. After adjusting for baseline clinicopathological and treatment factors, bone metastasis was the only endpoint in which PNI retained its prognostic significance (hazard ratio 1.42 , 95\% confidence interval 1.05$1.92, \mathrm{p}=0.021$ ).

Conclusions: The association between PNI and the development of bone metastases supports the inclusion of this parameter as a component of the routine histology report. Further this association suggests that evaluation of PNI may assist in selecting those patients who should be monitored more closely during follow-up.

Keywords: Prostate adenocarcinoma, perineural invasion, prognosis, metastases, ISUP grade.

\section{Introduction}

Infiltration of the perineural space of intraprostatic nerves has long been recognized as a feature of prostatic adenocarcinoma. ${ }^{1}$ Until relatively recently it was considered that this potential space offered less resistance to invasion by malignant cells and it was this that facilitated tumour spread beyond the prostate gland. ${ }^{2,3}$ More recently it has been recognized that perineural invasion (PNI) by tumour is a complex molecular process and it has been demonstrated to be associated with RET- associated cell transformation and activation of glial cell line - derived neurotrophic growth factor (GDNF) and its co-receptor GFR alpha1, which is expressed in nerves and reactive stromal cells. ${ }^{4}$

Multiple studies have investigated the prognostic significance of PNI by prostatic adenocarcinoma with conflicting results. These studies have employed a variety of methodologies, being based upon the detection of PNI in either diagnostic prostate biopsies, samples from transurethral resection of prostate or radical prostatectomy specimens. ${ }^{1,5-20}$ The outcome parameters for these studies are similarly variable and in most instances the presence of PNI was assessed against surrogate markers of tumour aggressiveness such as Gleason score/ ISUP grade, tumour stage, cell cycle activity, extraprostatic extension of tumour, seminal vesicle involvement, surgical margin positivity and the presence of lymph node metastases. The most frequently investigated outcome parameter was prostate specific 
antigen biochemical failure, while in some studies more meaningful outcome parameters (time to localized / systemic tumour progression, development of castration resistance, and cancer-specific and overall survival intervals) were available as clinical endpoints. In the majority of studies published to date, PNI was found not to correlate with these defined outcome parameters. ${ }^{5-7,10}$ Despite this some studies have shown compelling evidence that PNI has prognostic significance. Interestingly those studies based upon needle biopsies have more frequently shown the presence of PNI to correlate with tumour progression/ patient survival when compared with results obtained from analysis of radical prostatectomy specimens. ${ }^{1,5,10}$ In particular four separate studies have correlated the presence of PNI in needle biopsies with survival in patients treated by radical prostatectomy, external beam radiotherapy or a variety of modalities with the prognostic significance of PNI being retained on multivariable analysis. $^{14-17}$

In most published studies the patients have been managed with curative intent which may provide an uncontrolled variable, as potentially curable tumours may show a less aggressive clinical course. To date few studies have analysed the prognostic significance in patients managed, at least initially, by active surveillance and these have shown correlation between the presence of PNI and either tumour progression or cancer-related death. 10,19,20 While these studies do follow the natural clinical course of the disease - at least in its early stages - they also select patients whose disease is likely to have a less aggressive clinical course.

In our study we have investigated the significance of PNI as a prognostic parameter in men with locally advanced disease who were treated with radiotherapy and androgen deprivation and were followed as part of the TROG 03.04 RADAR randomised controlled trial. ${ }^{21,22}$

\section{Materials and Methods}

The cases in this study were accessed as the histological component of the TransTasman Radiation Oncology Group TROG 03.04 RADAR (Randomised Androgen Deprivation And Radiotherapy) trial. This is a phase 3 trial co-ordinated between Australia 
and New Zealand with 1071 subjects recruited from 23 treatment centres between October 2003 and August 2007.21,22

Eligibility criteria for enrolment in the trial were men $\geq 18$ years of age with histologically confirmed acinar adenocarcinoma of the prostate. They had no evidence of systemic metastases or lymph node involvement, with an Eastern Cooperative Oncology Group (ECOG) performance status of 0 or 1 and absence of concurrent disease that was likely to limit life expectancy to $<5$ years. Tumours were of clinical staging category cT $2 b$ to cT4, of any ISUP grade with baseline serum prostate-specific antigen (PSA) level, or clinical staging category $\mathrm{cT} 2 \mathrm{a}$ with ISUP grade $>1$ and baseline PSA of $\geq 10 \mathrm{ng} / \mathrm{mL}$.

Subjects were randomized to one of four groups in a $2 \times 2$ factorial study design: Group 1 - A control group of 6 months androgen suppression (AS) prior to radiotherapy (designated standard treatment, 6AS), Group 2 - Standard treatment plus 18 months zoledronic acid therapy (6AS $+\mathrm{Z}$ ), Group 3 - Standard treatment plus 12 months androgen suppression after radiotherapy (18AS) and Group 4 - Standard treatment plus 12 months androgen suppression after radiotherapy, and 18 months zoledronic acid therapy (18AS+Z). Radiotherapy to the prostate and seminal vesicles was administered according to an embedded dose escalation program. ${ }^{21,22}$

Diagnostic thin core biopsies were retrieved for all cases and reviewed blind by the Trial Pathologist (BD) in the post-randomisation phase of the trial during the period 2010 to 2015. The tumours were graded according to the recommendations of the International Society of Urological Pathology (ISUP) consensus conference and an ISUP grade was assigned to each case..$^{23,24}$ All cores and levels from each case were assessed for PNI by adenocarcinoma, and the total number of foci of PNI per case was recorded.

Participants were routinely followed up at 3 monthly intervals to 30 months, then 6 monthly to 60 months, then annually with PSA measures and clinical examinations. Investigations such as biopsy, CT scan, chest x-ray and bone scintigraphy were performed at the treating clinician's discretion. Ten year follow-up data from the time of randomisation were available for each case and were utilised in the time to event analysis. In our 10 year main endpoints report. global testing for interactions found no significant differences between the four treatment arms, ${ }^{25}$ hence arms could be collapsed to compare treatment factors separately. 


\section{Endpoints}

The primary endpoint for this study was bone metastasis. Secondary endpoints were soft tissue metastasis, transition to castration resistance, prostate cancer-specific mortality and all-cause mortality. Transition to castration resistance was a post-hoc endpoint (see Table S1 for definition). Investigations to diagnose metastases, including CT scans of the abdomen and pelvis, chest x-ray and isotopic whole-body bone scintigraphy, were mandated if symptoms suggested a need or if the PSA reached $20 \mathrm{ng} / \mathrm{mL}$. Death was attributed to prostate cancer if it occurred in the context of progressive metastatic disease or recurrent primary cancer causing urinary obstruction, without reasonable alternative unrelated causes. All endpoint imaging reports and causes of death were monitored at source and reviewed centrally by a group of senior clinician investigators, blinded to patient and treatment identity. Time-to-event endpoints were measured from randomization.

\section{Statistical Methods}

PNI was analysed as absent or present. Associations between PNI and clinicopathological variables were assessed using chi-square and Fisher's exact tests for categorical variables, and independent sample-t test and Wilcoxon rank sum tests for parametric and nonparametric tests, respectively. For each endpoint the Kaplan-Meier method and the log-rank test were used to compare failure rates according to PNI status. Univariable and multivariable Cox proportional hazards regression analyses were performed to obtain hazard ratios (HRs) with 95\% confidence intervals (CI). Multivariable models adjusted for patient age at randomisation (continuous); baseline PSA ( $<10$ vs 10-20 vs $>20 \mathrm{ng} / \mathrm{ml}$ ); ISUP grade (1-5); clinical tumour stage (T2 vs T3/T4); percent of biopsy cores positive for cancer (continuous); duration of androgen suppression (6AS vs 18AS), use of 18 months of zoledronic acid (no vs yes); and radiotherapy dose (66 Gy vs 70 Gy vs 74 Gy vs high dose rate brachytherapy). The proportional hazards assumption was tested by using Schoenfeld residuals. Covariates that violated the proportional hazards assumption were stratified for in these models. In a sensitivity analysis, models were repeated using competing risks regression and estimates for PNI effects were compared with those derived from Cox models. Competing risks were defined as death due to any cause for bone metastasis, soft tissue metastasis and transition to castration resistance, and death from other cause for prostate cancer-specific mortality. 
Analyses were performed on an intention-to-treat basis and a two-sided $\mathrm{p}$ value of $<0.05$ was considered statistically significant for all tests. Statistical analyses were programmed using Stata/IC Version 15.1.

\section{Results}

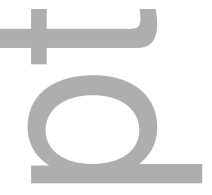

Histological material was available for PNI review from 976/1071 cases. The median age was 68.8 years (interquartile range [IQR], 63-73) and median follow-up was 10.6 years (IQR 8.3-11.8). PNI was detected in 46.0\% (449/976) cases, with 215 cases (22.0\%) having PNI in a single core. In the remaining 234 cases $(24.0 \%)$ there were 2 to 17 foci of PNI per case (Table 1).

A summary of clinicopathological characteristics according to PNI status is shown in Table 2. The presence of PNI was significantly associated with higher ISUP grade, stage and National Comprehensive Cancer Network risk group, as well as number of biopsy cores taken and percentage of positive cores. No association was found between PNI and patient age or baseline PSA. There were no significant imbalances in allocation to treatment arm; however, patients with PNI present were more likely to receive a higher radiotherapy dose. Table 3 gives a more detailed breakdown of the distribution of ISUP grade and CT staging category according to PNI status.

The number of endpoint events by PNI status is presented in Table 4 and the effect of PNI on outcomes in uni- and multivariable Cox regression models is summarised in Table 5. During the follow-up period, 212/976 (21.7\%) patients developed bone metastases. Fig. 1 depicts the Kaplan-Meier curve for cumulative probability of bone metastasis according to PNI status. On univariable analysis the presence of PNI was significantly associated with bone metastases, hazard ratio (HR) 2.09, 95\% confidence interval (CI) 1.58-2.75, $\mathrm{p}<0.0001$. The adverse impact of PNI was confirmed in the adjusted model (HR 1.42, 95\% CI 1.051.92, $\mathrm{p}=0.021$ ) (Table 6). For all secondary endpoints, PNI was a significant prognostic factor in univariable analyses, however did not retain its statistical significance in the adjusted models. Full results of the multivariable analyses for secondary endpoints are presented in Table S2. In sensitivity analyses, estimates for PNI effects derived from competing risks models were similar to those from Cox models (Table S3). 


\section{Discussion}

The prevalence of PNI in prostate biopsies varies between studies, being present in up to $34 \%$ of cases. ${ }^{14}$ In large series the prevalence is in the order of $20 \%, 5,6,10$ which contrasts with our own findings of PNI in $46 \%$ of biopsies. If sections are not subjected to formal review by a specialist genitourinary pathologist and data are based upon the descriptions contained in the original histological reports, then it is likely that PNI will be overlooked and therefore be under-represented. This appears to be most striking in studies relating to PNI in radical prostatectomy specimens. In cases where PNI was detected utilizing routine haematoxylin and eosin sections the prevalence ranged from $2.8 \%$ to $84.5 \%{ }^{18,26}$ This contrasts with our earlier study where PNI was identified through immunostaining for S-100 protein and was observed in $90 \%$ of cases. ${ }^{1}$

It is clear that, if searched for carefully, PNI is usually present in radical prostatectomy specimens. A needle biopsy only samples a small proportion of the prostate gland and this most likely accounts for the apparent discrepancy in the prevalence of PNI between needle biopsy and radical prostatectomy specimens. Despite these sampling issues needle biopsies usually sample the peripheral zone of the prostate while targeted biopsies focus on the tumour and surrounding tissue. In view of this it is understandable that any PNI detected in these specimens is going to be of greater potential prognostic significance as a marker of extraprostatic spread of tumour than foci of PNI within a radical prostatectomy specimen that are situated some distance from the prostatic margin.

In most reported studies that have investigated the predictive prognostic significance of PNI, patients had received therapy with curative intent, consisting of radical prostatectomy or external beam radiation. Patients enrolled in the RADAR trial presented with locally advanced disease and this has provided a rare opportunity to monitor the natural history of the disease and assess potential prognostic parameters. All biopsies were subjected to blind review by a urological pathologist and patients were followed for at least 10 years or until death. During the follow-up period evidence of local tumour progression, and the development of metastatic disease was monitored. ${ }^{21,22}$ This study has demonstrated that PNI was associated with the development of bone metastases. 
Four previous studies have investigated the prognostic significance of PNI in biopsies from patients who were not treated with curative intent. ${ }^{10,11,19,20}$ In two of these studies patients underwent active surveillance and the presence of PNI was correlated with tumour progression. ${ }^{19,20}$ In a separate study a cohort of patients diagnosed by transurethral resection of prostate were enrolled in a watchful waiting programme and PNI was correlated with cancer-specific death on univariable analysis, but not on multivariable analysis. ${ }^{10}$ Most recently Ahmad et al. investigated the association of PNI with cancer-specific survival in 998 patients identified through cancer registries who had localized disease and had not received potentially curative therapy for at least the first 6 months post-diagnosis. ${ }^{11}$ While there was a significant association between outcome and the presence of PNI on univariable analysis, this was lost when grade, stage, tumour volume and serum PSA were added to the analysis. In that study no other outcome parameter beyond cause-specific death was considered and this may have influenced the conclusions. Prostate cancer typically has a prolonged clinical course and this study does not report the number of patients who developed recurrent or progressive disease by the termination of follow-up - which for some patients was after 9 years. In the 10 year follow-up of the RADAR trial $21.7 \%$ of men developed bone metastases, $17.5 \%$ developed soft tissue metastases, $15.4 \%$ showed transition to castration resistance, and $35 \%$ of patients died, with $38 \%$ of these deaths attributed to prostate cancer. PNI was associated with all of the markers of disease progression on univariable analysis. However, after adjusting for baseline clinicopathological and treatment factors, bone metastasis was the only endpoint in which PNI retained its prognostic significance.

Bone metastases are a common feature of advanced prostate adenocarcinoma and the association with PNI is of some interest. In a study of 663 patients with prostate cancer on biopsy and evidence of 'high risk' disease (existing symptoms, Gleason score $\geq 8$, cT3+ disease or cT1 tumours with a PSA $>20 \mathrm{ng} / \mathrm{ml}$ ) the relationship between co-existing bone metastases and PNI was assessed. ${ }^{7}$ These patients underwent bone scan and $46.9 \%$ were shown to have bone metastases at the time of presentation. The presence of PNI correlated with the presence of bone metastases and this was independent of serum PSA levels, Gleason score and clinical stage. The study showed that PNI was a predictor of concurrent bone metastases, while our study demonstrated that the presence of PNI at the time of diagnosis also predicts the temporal development of bone metastases. 
In terms of cellular and molecular mechanisms, at this stage it is unclear why a local process such as PNI is associated with metastasis. The classic interpretation for the association between PNI and cancer prognosis in general is that PNI is a marker of cancer cell invasiveness. ${ }^{6}$ Recent evidence from animal models has demonstrated the importance of the role of nerve-cancer cell crosstalk in prostate cancer. ${ }^{27}$ In the mouse, denervation of autonomic nerves prevents prostate cancer growth and dissemination, and although the mechanism has yet to be fully elucidated, this involves the stimulation of beta-adrenergic and muscarinic receptors in both cancer and stromal cells contributing to the stimulation of prostate cancer growth and metastasis. ${ }^{28,29}$ PNI results from a bi-directional interaction between nerves and cancer cells and the presence of PNI may indicate active nerve-cancer cell crosstalk, which could promote tumour aggressiveness. However, additional functional investigations are warranted to clarify the molecular basis for the association between PNI and metastasis in prostate cancer.

In conclusion, PNI in the RADAR trial was significantly associated with the development of bone metastases in both uni- and multivariable analyses. This association supports the inclusion of this parameter as a component of the routine histological reporting of prostate cancer in needle biopsies.

\section{Acknowledgements}

$\mathrm{BD}$, JD and AS designed the study and BD and AS wrote the manuscript. All authors contributed to data collection/interpretation and commented on the final draft.

\section{References}
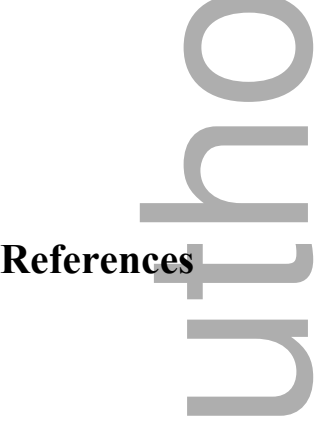

1. Merrilees AD, Bethwaite PB, Russell GL, Robinson RG, Delahunt B. Parameters of perineural invasion in radical prostatectomy specimens lack prognostic significance. Mod Pathol 2008; 21: 1095-1100.

2. Akert K, Sandri C, Weibel ER, Peper K, Moor H. The fine structure of the perineural endothelium. Cell tissue Res 1976; 164: 281-295. 
3. Hassan MO, Maksem J, The prostatic perineural space and its relation to tumor spread: an ultrastructural study. Am J Surg Pathol 1980; 4: 143-148.

4. Ban K, Feng S, Shao L, Ittman M. RET signalling in prostate cancer. Clin Cancer Res 2017; 23: 4885-4896.

5. Harnden P, Shelley MD, Clements $\mathrm{H}$ et al. The prognostic significance of perineural invasion in prostatic cancer biopsies: a systematic review. Cancer 2007; 107: $13-24$.

6. Leibig C, Ayala G, Wilks JA, Berger DH, Albo D. Perineural invasion in cancer: a review of the literature. Cancer 2009: 115; 3379-3391.

7. Ciftci S, Yilmaz H, Ciftci E et al. Perineural invasion in prostate biopsy specimens is associated with increased bone metastases in prostate cancer. The Prostate 2015; 75: 1783-1789.

8. Sæter T, Bogaard M, Vlatkovic L et al. The relationship between perineural invasion, tumour grade, reactive stroma, and prostate cancer-specific mortality: a clinicopathologic study on a population-based cohort. The Prostate 2016; 76: 207214.

9. Truong M, Rais-Bahrami S, Nix YW et al. Perineural invasion by prostate cancer on MR/ US fusio targeted biopsy is associated with extraprostatic extension and early biochemical recurrence after radical prostatectomy. Hum Pathol 2017; 66: 206-211.

10. Zareba $\mathrm{P}$, Flavin R, Isikbay $\mathrm{M}$ et al. Perineural invasion and risk of lethal prostate cancer. Cancer Epidemiol Biomarkers Prev 2017; 26: 719-726.

11. Ahmed AS, Parameshwaran V, Beltran L et al. Should reporting of peri-neural inyasion and extra-prostatic extension be mandatory in prostate cancer biopsies. Correlation with outcome in biopsy cases treated conservatively. Oncotarget 2018; 9: 20555-20562.

12. Lubig S, Thiesler T, Müller S et al. Quantitative perineural invasion is a prognostic marker in prostate cancer. Pathology 2018; 50: 298-304.

13. Beard C, Schultz D, Loffredo M et al. Perineural invasion associated with increased cancer-specific mortality after external beam radiation therapy for men with lowand intermediate-risk prostate cancer. J Urol 2001; 165: 126-129.

14. Feng FY, Qian Y, Stenmark MH et al. Perineural invasion predicts increased recurrence, metastases, and death from prostate cancer following treatment with dose-escalated radiation therapy. Int J Radiat Oncol Biol Phys 2011; 81: e361-7. 
15. Delancy JO, Wood DP Jr, He C et al. Evidence of perineural invasion on prostate biopsy specimen and survival after radical prostatectomy. Urology 2013; 81: 354357.

16. Tollefson MK, Karnes RJ, Kwon ED et al. Prostate cancer Ki-67 (MIB-1) expression, perineural invasion and Gleason score as biopsy-based predictors of prostate cancer mortality: the Mayo model. Mayo Clin Proc 2014; 89: 308-318.

17. De La Taille A, Rubin MA, Bagiella E et al. Can perineural invasion on prostate needle biopsy predict prostate specific androgen recurrence after radical prostatectomy. J Urol 1999; 162: 103-106.

18. Kozal S, Peyronnet B, Catterino S et al. Influence of pathological features on oncological outcomes after robot-assisted radical prostatectomy for localized prostate cancer: results of a prospective study. Urol Oncol 2015; 33: 330e1-330e7

19. Cohn JA, Dangle PP, Wag CE et al. The prognostic significance of perineural invasion and race in men considering active surveillance. BJU Int 2014; 114: 75-80.

20. Moreira DM, Fleshner NE, Freedland SJ. Baseline perineural invasion is associated with shorter time to progression in men with prostate cancer undergoing active surveillance: results from the REDEEM study. J Urol 2015; 194: 1258-1263.

21. Denham JW, Joseph D, Lamb DS, et al. Short-term androgen suppression and radiotherapy versus intermediate-term androgen suppression and radiotherapy, with or without zoledronic acid, in men with locally advanced prostate cancer (TROG 03.04 RADAR): an open-label, randomised phase 3 factorial trial. Lancet Oncol $2014 ; 15 ; 1076-89$.

22. Denham JW, Steigler A, Joseph D, et al. Radiation dose escalation or longer androgen suppression for locally advanced prostate cancer? Data from the TROG 03.04 RADAR trial. Radiother Oncol 2015; 115; 301-307.

23. Epstein JI, Egevad L, Amin MB et al. The 2014 International Society of Urological Pathology (ISUP) consensus conference of Gleason grading of prostatic carcinoma. Definition of grading pattern and proposal for a new grading system. Am J Surg Pathol 2016; 40: 244-252.

24. Delahunt B, Egevad L, Srigley JR et al. Validation of International Society of Urological Pathology (ISUP) grading for prostatic adenocarcinoma in thin core biopsies using TROG 03.04 'RADAR' trial clinical data. Pathology 2015; 47; 520525. 
25. Denham JW, Joseph D, Lamb DS, et al. Short-term androgen suppression and radiotherapy versus intermediate-term androgen suppression and radiotherapy, with or without zoledronic acid, in men with locally advanced prostate cancer (TROG 03.04 RADAR): 10-year results from a randomised, phase 3, factorial trial. Lancet Oncol 2019; 20: 267-281.

26. Ost P, De Troyer B, Fonteyne V et al. A matched control analysis of adjuvant and salvage high-dose postoperative intensity-modulated radiotherapy for prostate cancer. Int J Radiat Oncol Biol Phys 2011; 80: 1316-1322.

27. March B, Faulkner S, Jobling $\mathrm{P}$ et al. Tumour innervation and neurosignalling in prostate cancer. Nat Rev Urol 2020;17: 119-130.

28. Magnon C, Hall SJ, Lin J et al. Autonomic nerve development contributes to prostate cancer progression. Science 2013; 341: 1236361.

29. Zahalka AH, Arnal-Estapé A, Maryanovich M et al. Adrenergic nerves activate an angio-metabolic switch in prostate cancer. Science 2017; 358: 321-326. 
Table 1. Frequency distribution of number of cores with PNI

\begin{tabular}{|cc}
\hline Number of cores with & Number of cases \\
\hline 1 & 215 \\
2 & 118 \\
3 & 58 \\
4 & 29 \\
5 & 10 \\
6 & 9 \\
7 & 5 \\
8 & 2 \\
9 & 1 \\
10 & 1 \\
17 & 1 \\
\hline
\end{tabular}




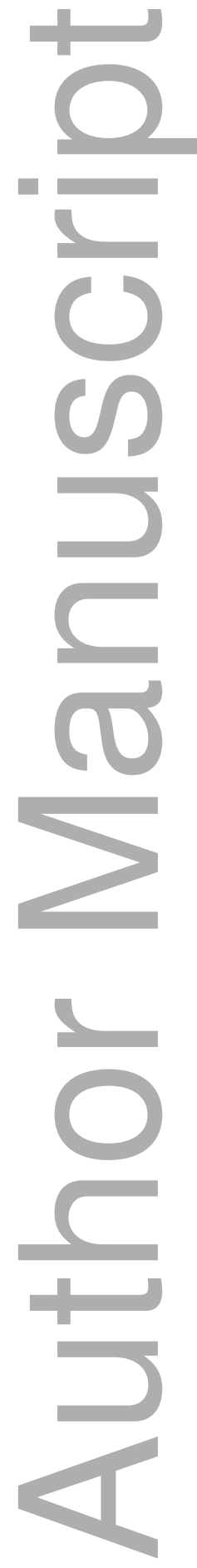

This article is protected by copyright. All rights reserved 
Table 2. Clinicopathological characteristics of patients by PNI status ( $n=976$ )

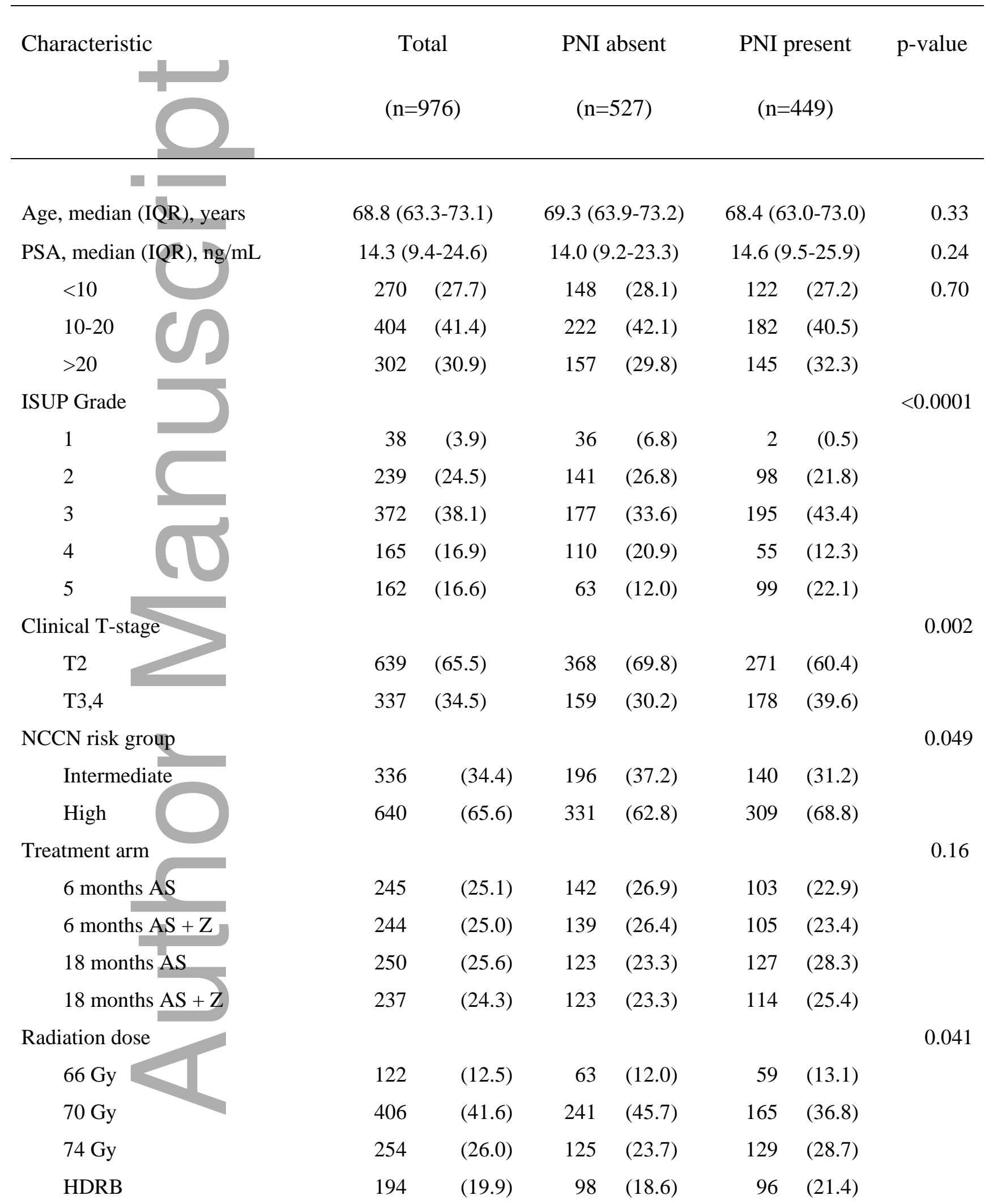

Biopsy cores, median (IQR)

This article is protected by copyright. All rights reserved 
Number taken

Percent positive
$9(7-12)$

57 (38-83)
$9(7-12)$

50 (29-67)
$10(7-12)$

0.002

$69(50-91) \quad<0.0001$

Data are $\mathrm{n}(\%)$ unless otherwise stated

Percentages may not total 100 due to rounding

Abbreviations: PNI, perineural invasion; IQR, interquartile range; PSA, prostate-specific antigen; ISUP, International Society of Urological Pathology; NCCN, National Comprehensive Cancer Network; AS, androgen suppression; Z, zoledronic acid; Gy, Gray; HDRB, high dose rate brachytherapy

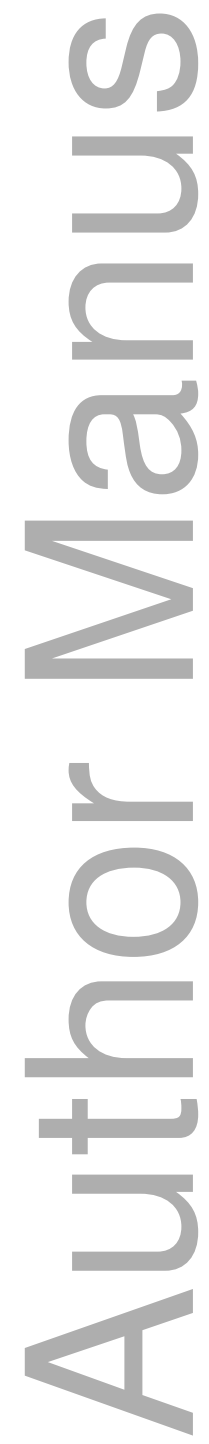

This article is protected by copyright. All rights reserved 


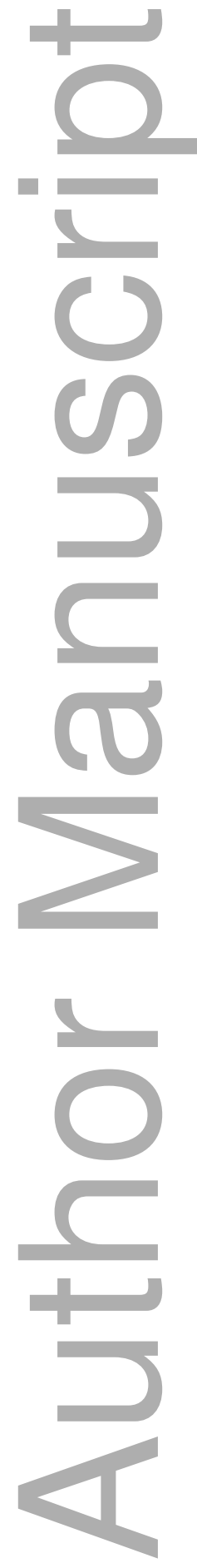

This article is protected by copyright. All rights reserved 
Table 3. cT staging category and ISUP grade according to PNI status

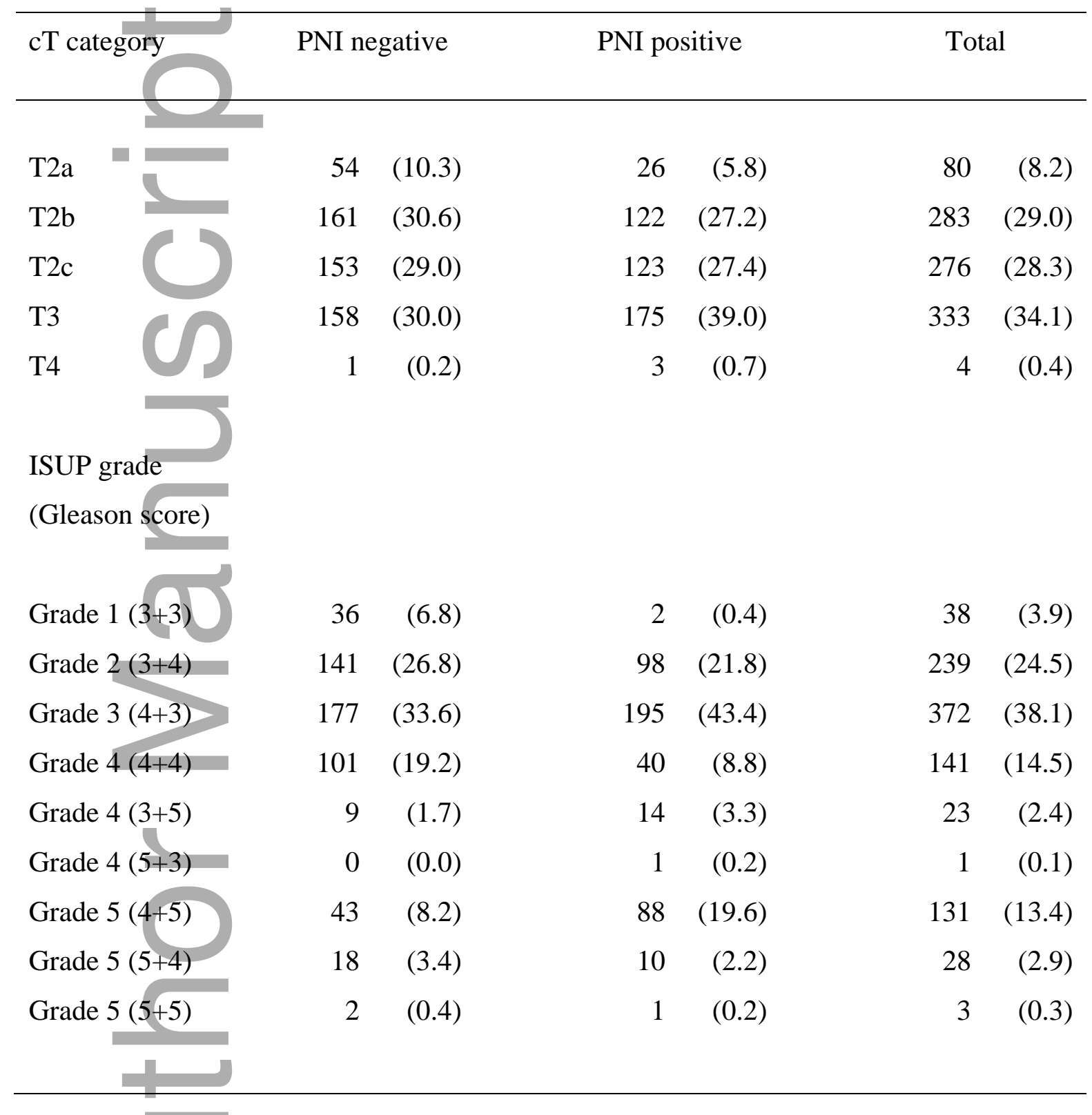

Data are $\mathrm{n}(\%)$

Percentages may not total 100 due to rounding

Abbreviations: PNI, perineural invasion; ISUP, International Society of Urological Pathology 
Table 4. Number of endpoint events by PNI status

\begin{tabular}{lcccccc}
\hline & $\begin{array}{c}\text { PNI absent } \\
(\mathrm{n}=527)\end{array}$ & $\begin{array}{c}\text { PNI present } \\
(\mathrm{n}=449)\end{array}$ & $\begin{array}{c}\text { Total } \\
(\mathrm{n}=976)\end{array}$ \\
$\begin{array}{l}\text { Bone metastasis } \\
\text { Soft tissue metastasis }\end{array}$ & 82 & $(15.6)$ & 130 & $(29.0)$ & 212 & $(21.7)$ \\
Transition to castration resistance & 64 & $(12.1)$ & 86 & $(19.2)$ & 150 & $(15.4)$ \\
Prostate cancer death & 53 & $(10.1)$ & 77 & $(17.2)$ & 130 & $(13.3)$ \\
Death from any cause & 168 & $(31.9)$ & 176 & $(39.2)$ & 344 & $(35.3)$ \\
& & & & & &
\end{tabular}

Data are n $(\%)$

Abbreviations: PNI, perineural invasion

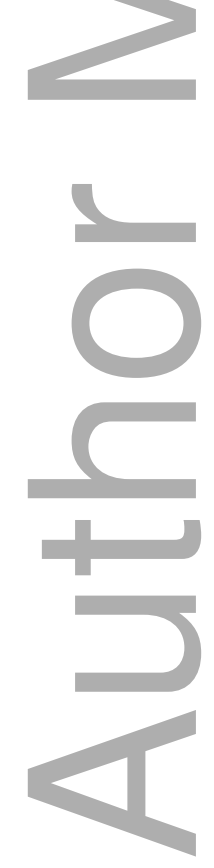

This article is protected by copyright. All rights reserved 
Table 5. Effect of PNI on prostate cancer progression and mortality endpoints

\begin{tabular}{|c|c|c|c|c|c|c|}
\hline \multirow[b]{2}{*}{ Endpoint } & \multicolumn{3}{|c|}{ Univariable } & \multicolumn{3}{|c|}{ Multivariable* } \\
\hline & HR & $95 \% \mathrm{CI}$ & $\mathrm{p}$ value & HR & $95 \% \mathrm{CI}$ & $\mathrm{p}$ value \\
\hline Bone metastasis & 2.09 & $(1.58-2.75)$ & $<0.0001$ & 1.42 & $(1.05-1.92)$ & 0.021 \\
\hline Soft tissue metastasis & 1.46 & $(1.08-1.97)$ & 0.014 & 0.90 & $(0.65-1.25)$ & 0.54 \\
\hline $\begin{array}{l}\text { Transition to castratio } \\
\text { resistance }\end{array}$ & 1.71 & $(1.23-2.36)$ & 0.001 & 1.12 & $(0.79-1.59)$ & 0.52 \\
\hline $\begin{array}{l}\text { Prostate cancer-specif } \\
\text { mortality }\end{array}$ & 1.85 & $(1.30-2.62)$ & 0.0006 & 1.10 & $(0.75-1.60)$ & 0.63 \\
\hline All-cause mortality & 1.32 & $(1.06-1.63)$ & 0.011 & 1.03 & $(0.82-1.29)$ & 0.82 \\
\hline
\end{tabular}

Abbreviations: PNI, perineural invasion; HR, hazard ratio; CI, confidence interval

*Multivariable models adjusted for patient age at randomisation (continuous); baseline PSA (<10 vs 10-20 vs >20 ng/ml); ISUP grade (1-5); tumour stage (T2 vs T3/T4); percent positive biopsy cores (continuous); duration of androgen suppression (6AS vs 18AS); use of 18 months of zoledronic acid (no vs yes); and radiotherapy dose (66 Gy vs 70 Gy vs 74 Gy vs high dose rate brachytherapy)

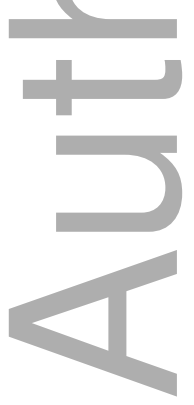


Table 6. Multivariable analysis for bone progression

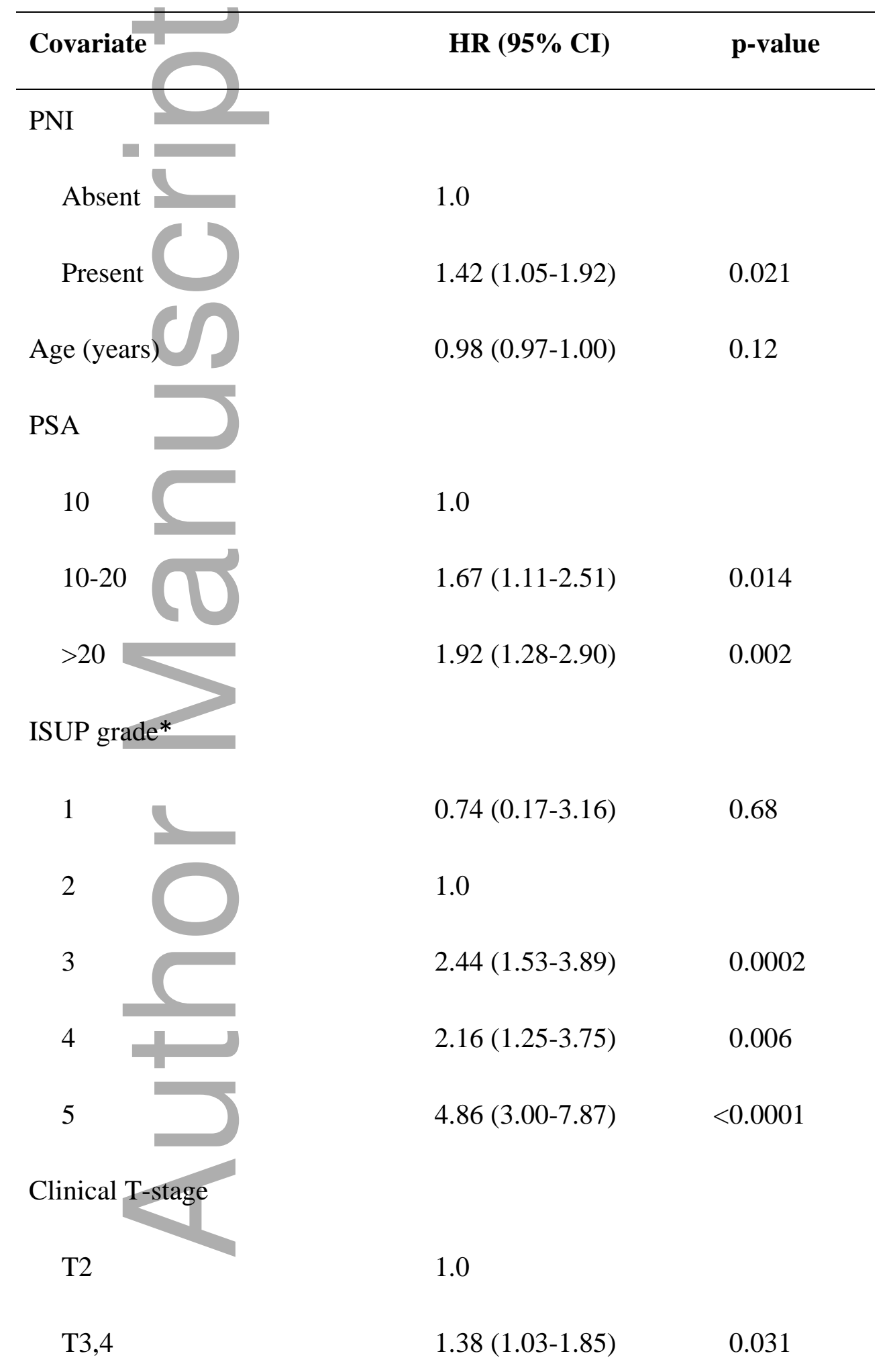




\begin{tabular}{|c|c|c|}
\hline Covariate & HR $(95 \% \mathrm{CI})$ & p-value \\
\hline Percent positive biopsy cores & $1.01(1.01-1.02)$ & 0.0001 \\
\hline \multicolumn{3}{|l|}{ AS duration } \\
\hline 6 montl & 1.0 & \\
\hline 18 months & $0.58(0.44-0.77)$ & 0.0001 \\
\hline \multicolumn{3}{|l|}{ Zoledronic acid } \\
\hline No & 1.0 & \\
\hline Yes & $1.02(0.78-1.34)$ & 0.89 \\
\hline \multicolumn{3}{|l|}{ Radiation dose* } \\
\hline $66 \mathrm{~Gy}$ & $0.95(0.62-1.45)$ & 0.80 \\
\hline 70 Gy & 1.0 & \\
\hline 74 Gy & $0.80(0.56-1.13)$ & 0.21 \\
\hline HDRB & $0.71(0.48-1.04)$ & 0.08 \\
\hline
\end{tabular}

Abbreviations: HR, hazard ratio; CI, confidence interval; PNI, perineural invasion; PSA, prostate-specific antigen; ISUP, International Society of Urological Pathology; AS, androgen suppression; Gy, Gray; HDRB, high dose rate brachytherapy

* ISUP grade 2 and 70 Gy were selected as reference levels due to the relatively small number of participants in the subgroups for ISUP grade 1 and 66 Gy 
Figure 1. Cumulative probability of bone metastasis by PNI status
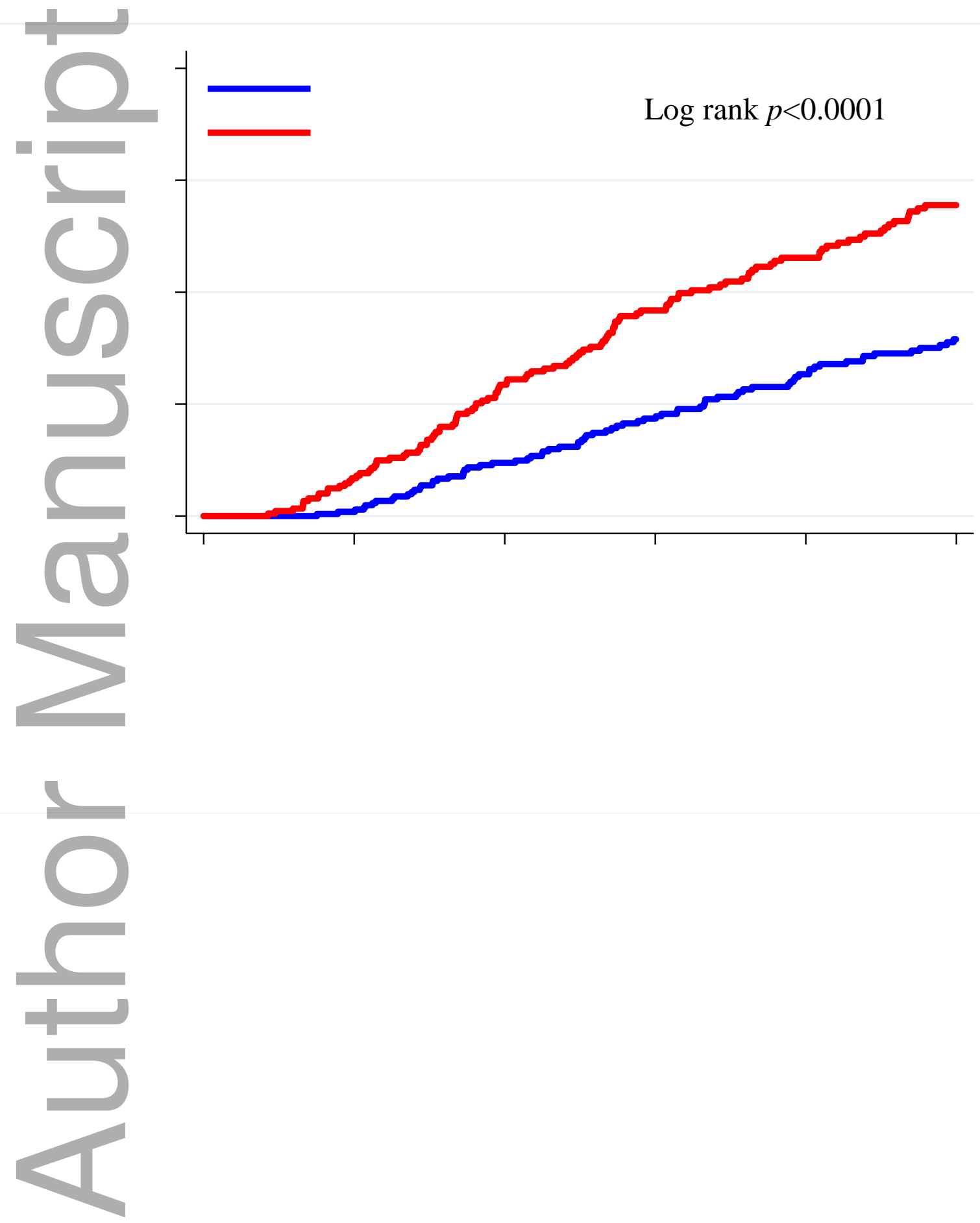

This article is protected by copyright. All rights reserved 


\section{University Library}

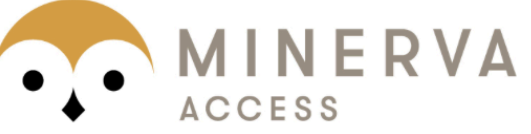

A gateway to Melbourne's research publications

Minerva Access is the Institutional Repository of The University of Melbourne

\section{Author/s:}

Delahunt, B;Murray, JD;Steigler, A;Atkinson, C;Christie, D;Duchesne, G;Egevad, L;Joseph,

D;Matthews, J;Oldmeadow, C;Samaratunga, H;Spry, NA;Srigley, JR;Hondermarck,

$\mathrm{H}$;Denham, JW

Title:

Perineural invasion by prostate adenocarcinoma in needle biopsies predicts bone metastasis: Ten year data from the TROG 03.04 RADAR Trial

\section{Date:}

2020-06-18

\section{Citation:}

Delahunt, B., Murray, J. D., Steigler, A., Atkinson, C., Christie, D., Duchesne, G., Egevad, L., Joseph, D., Matthews, J., Oldmeadow, C., Samaratunga, H., Spry, N. A., Srigley, J. R., Hondermarck, H. \& Denham, J. W. (2020). Perineural invasion by prostate adenocarcinoma in needle biopsies predicts bone metastasis: Ten year data from the TROG 03.04 RADAR Trial. HISTOPATHOLOGY, 77 (2), pp.284-292. https://doi.org/10.1111/his.14107.

Persistent Link:

http://hdl.handle.net/11343/275927 